\title{
New signal processing for fast and precise QEPAS measurements
}

\author{
Raphael Levy \\ DPHY \\ ONERA- The French Aerospace \\ Lab \\ Chatillon, France \\ raphael.levy@onera.fr
}

\author{
Maxime Duquesnoy \\ MIRSENSE \\ Palaiseau, France
}

Guillaume Aoust
MIRSENSE
Palaiseau, France
Jean Michel Melkonian DPHY

ONERA- The French Aerospace $\mathrm{Lab}$

Palaiseau, France

\author{
Myriam Raybaut \\ DPHY \\ ONERA- The French Aerospace Lab \\ Palaiseau, France
}

\begin{abstract}
Quartz Enhanced Photoacoustic Spectroscopy (QEPAS) gas sensors have been widely developed over the last decade. This technique takes advantage of a high quality factor tuning fork to enable high sensitivity and high selectivity miniature gas sensors. Lock-in detection is classically used to measure the resonator amplitude which is proportional to the gas concentration, but this technique is slow and leads to measurement drifts as it does not follow the resonator frequency drifts over temperature and pressure. This paper presents a new QEPAS signal processing technique that allows faster and more stable measurements that will enable accurate and fast multi gas sensors.
\end{abstract}

Keywords-QEPAS, quartz, resonator, photoacoustic, spectroscopy

\section{INTRODUCTION}

Photo-acoustic spectroscopy is now a well-established technique in which a gas is detected through the sound it generates by non-radiative relaxation consecutive to absorption of modulated light at a wavelength matching a vibrational transition [1] or a rotational transition [2]. In traditional photoacoustic spectroscopy the sound pressure is amplified by an acoustic cavity and detected by a microphone. In QuartzEnhanced Photo-Acoustic Spectroscopy (QEPAS), the sound pressure is generated between the prongs of a quartz tuning fork which actuates it and generates an electrical signal through quartz piezo-electric transduction.

QEPAS sensors have been widely studied since the first publication in 2002 [3], analytical models of the sensor have been developed $[4,5]$ as well as optimized resonant detectors coupled to micro tubes acoustic resonators in different configurations [6,7], but signal processing has not been studied. Usually, lock-in detection, as shown in figure 1 , is used to extract the signal at a given frequency from the surrounding noise within a reduced bandwidth. However this detection scheme shows a major drawback that is slow measurement, preventing QEPAS to be used for rapid and multi-gas sensing (measurement time $<100 \mathrm{~ms}$ ).

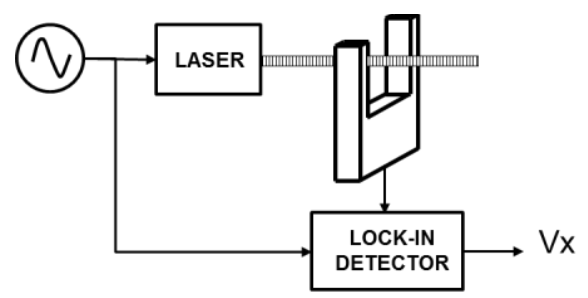

Figure 1: QEPAS lock-in detection scheme.

Indeed, in the frame of lock-in detection, the resonator is not actively controlled at resonance, and as the resonance shifts over temperature, pressure or aging, a sweep of the laser modulation frequency must be performed to detect the frequency that corresponds to the resonator maximum displacement. For each frequency point, the measurement time corresponds to:

$$
\tau=\frac{Q}{\pi f_{r}}
$$

where $Q$ is the resonator quality factor, and $f_{r}$ its eigen frequency.

This measurement time can be as long as a few tens of seconds as Q must be high $(>10000)$ and $\mathrm{f}_{\mathrm{r}}$ low $(<35 \mathrm{kHz})$ to achieve high sensitivity [3]. This long measurement time also decreases accuracy as measurements integrate the signal drifts. Thus it hinders high speed measurement of gas with no idle time.

Only a few other detection schemes were presented to our best knowledge. A technique called electrical modulation cancellation method [8] was used to outbalance the background noise induced by the illumination of the QTF prongs by the laser source. Another method consists in actuating the QTF with optical actuation as well as electrical actuation at the same time [9], while the resonator displacement is still measured with a lock-in detector: this enables to keep the resonator at resonance or to measure periodically the resonator Q factor. Yet another technique consists in using a beat frequency to obtain fast measurements, down to the millisecond, of the sensor quality factor, resonance frequency as well as gas concentration [10]. 
In this paper, we propose a closed loop detection scheme enabling fast and precise measurements as well as background noise cancellation. Its theoretical analysis is presented in the following sections as well as first experimental results.

\section{THEORETICAL ANALYSIS OF QEPAS CLOSED LOOP DETECTION}

\section{A. Principle of operation}

The principle of operation shown in Fig. 2 is as follows: the tuning fork is actuated at resonance by means of an oscillator circuit, the oscillator output signal is phase shifted and used to modulate the laser intensity. The laser beam passes in between the tuning fork prongs, generating a photoacoustic signal at the resonator eigen frequency. A phase quadrature is set between the resonator piezoelectric actuation force from the oscillator circuit and the photoacoustic actuation force from the laser beam. This induces a shift of the oscillator frequency that is proportional to the photoacoustic force and thus to the gas concentration. This frequency shift is instantaneous allowing fast measurements.

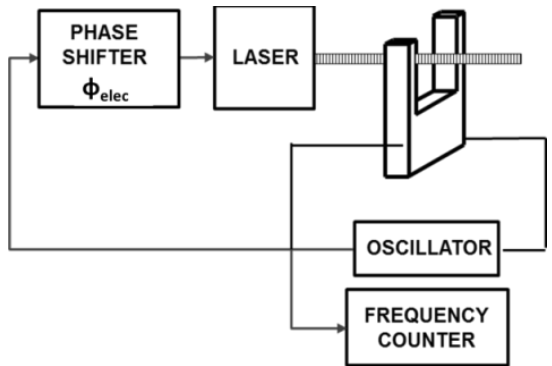

Fig. 2. QEPAS active detection scheme. The resonator oscillations are sustained by means of an oscillator circuit. The oscillator output signal is phase shifted and controls the modulation of the laser intensity. The measured frequency shift is proportional to the resonator vibration amplitude.

\section{B. Theoretical model}

The closed loop detection scheme is presented in Fig. 2, its equivalent block scheme is shown in Fig. 3. Two feedback branches contribute to actuate the resonator: the oscillator circuit branch that leads to the piezoelectric actuation force $\mathrm{F}_{\mathrm{pz}}$, and the photoacoustic branch that leads to the photoacoustic force $\mathrm{F}_{\mathrm{pa}}$. In order to obtain an oscillator frequency shift depending on the photoacoustic force, these two forces must be in phase quadrature. This is performed by adjusting the phase shift $\Phi_{\text {elec }}$ through the phase shifter circuit. Thus, the two modulated forces are in phase quadrature if:

$$
\frac{\pi}{2}=\Phi_{\text {pa }}+\Phi_{\text {propagation }}+\Phi_{\text {elec }} .
$$

$\Phi_{\mathrm{pa}}$ is the photoacoustic phase shift between the laser modulation and the force on the resonator prongs [3]:

$$
\Phi_{\mathrm{pa}}=\frac{\pi}{2}-\operatorname{atan}\left(2 \pi f_{r} \tau_{V T}\right)
$$

where $f_{r}$ is the modulation frequency, $\tau_{v t}$ the vibrationaltranslational relaxation time of the gas, depending on the wavelength and the gas mixture. $\Phi_{\text {propagation }}$ is the acoustic propagation delay from the laser beam to the resonator prongs.
Assuming there is no acoustic radiation from the resonator, it can be expressed as:

$$
\Phi_{\text {propagation }}=\frac{2 \pi \mathrm{L}}{\lambda_{a}}
$$

where $\mathrm{L}$ is the distance from the beam to the resonator branch, and $\lambda_{a}$ the acoustic modulation wavelength. For our custom tuning fork described in section III, this phase shift is: $\Phi_{\text {propagation }}=0.4 \mathrm{rad}$.

If the two actuation forces act in phase quadrature, the total force actuating the resonator is:

$$
\begin{gathered}
F_{\text {tot }}=\sqrt{\left|F_{p z}\right|^{2}+\left|F_{p a}\right|^{2}} \sin \left(2 \pi f_{r} t+\theta\right) \\
\theta=\operatorname{atan}\left(\frac{F_{\mathrm{pa}}}{\left|F_{\mathrm{pz}}\right|}\right),
\end{gathered}
$$

where $\theta$ is the additional phase shift in the oscillator loop induced by the two parallel feedback loops (see Fig. 3). This additional phase shift in the oscillator loop induces an oscillator frequency shift:

$$
\Delta f_{r}=\frac{\theta}{2 Q} f_{r}
$$

Considering that the photoacoustic force is order of magnitudes smaller than the piezoelectric force, we have:

$$
\Delta f_{r}=\frac{1}{2 Q} f_{r} \frac{F_{\mathrm{pa}}}{\left|F_{\mathrm{pz}}\right|}
$$

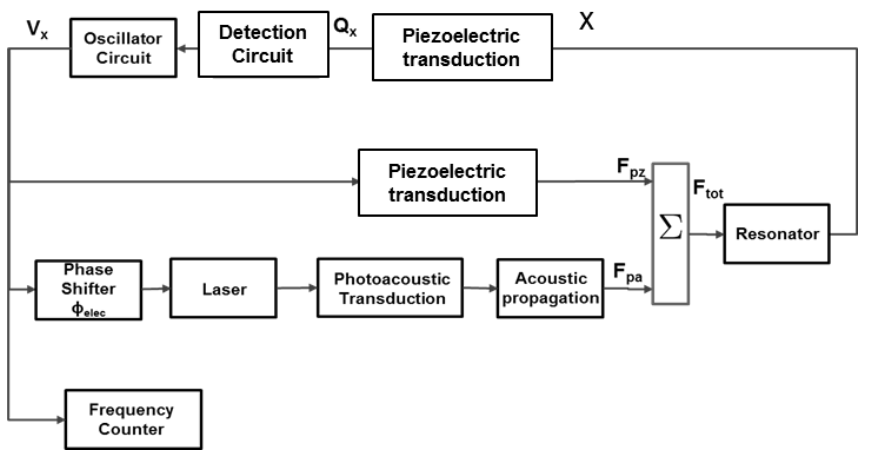

Fig. 3. Closed loop detection scheme. The oscillator feedback circuit branch is presented as well as the photoacoustic feedback. The oscillator frequency shift, proportional to the resonator displacement, is measured by means of the frequency counter.

\section{Differential Measurements enabled}

The active detection scheme described above also makes it possible to remove the influence of resonator eigen frequency variations over temperature by periodically adding a $\pi$ phase shift through the phase shifter circuit. Indeed a $\pi$ additional phase shift inverts the sign of the acoustic force $F_{p a}$ applied to the resonator, and thus inverts the sign of the oscillator frequency variation (see Eqs. $6 \&$ 7). Let us write the frequency of the oscillator without supplementary phase shift is:

$$
\left.f_{r}\right|_{0}=f_{r 0}+\Delta f_{r}+\Delta f_{r}(T)
$$


where $\mathrm{f}_{\mathrm{r} 0}$ is the initial eigen frequency of the resonator, $\Delta f_{r}$ is the photoacoustic induced frequency variation, $\Delta f_{r}(T)$ is the temperature induced frequency variation. The frequency of the oscillator with a $\pi$ supplementary phase shift is:

$$
\left.f_{r}\right|_{\pi}=f_{r 0}-\Delta f_{r}+\Delta f_{r}(T) .
$$

The frequency variation over temperature is then cancelled by switching the additional phase shift between 0 and $\pi$ and subtracting the two measured frequencies:

$$
\left.f_{r}\right|_{0}-\left.f_{r}\right|_{\pi}=2 \Delta f_{r}
$$

\section{Limitation of the technique}

The addition of a photoacoustic actuation force in phase quadrature with the piezoelectric force causes a phase shift in the oscillator loop. This phase shift must remain low enough for the oscillation conditions to remain valid, i.e. it must remain in the resonator bandwidth:

$$
\Delta f_{r} \ll \frac{f_{r}}{Q} .
$$

This is the case in the experiment detailed in section III; considering $1 \%$ concentration of $\mathrm{C}_{2} \mathrm{H}_{2}$ diluted in air and the experimental set-up described; $\Delta f_{r}=8 \mathrm{mHz}$, and $\frac{f_{r}}{Q}=2.6 \mathrm{~Hz}$ as shown in Fig. 9 and Table 1. Considering (8), this implies that the photoacoustic force is more than two orders of magnitude lower than the piezoelectric force.

\section{E. Noise calculation}

In order to compare the resolution of lock-in open loop detection and closed loop detection, the force equivalent noise is calculated for both detection techniques.

\section{1) Lock-in Detection Noise}

It has been shown that the ultimate QEPAS noise source originates from the resonator Brownian noise, provided that the system is used near resonance and that the electronical scheme has been correctly designed [4]. The white Brownian noise power spectral density can be expressed as:

$$
F_{\text {open loop }}=4 k T \frac{M 2 \pi f_{r}}{Q}\left(\mathrm{~N}^{2} / \mathrm{Hz}\right),
$$

where $k$ is the Boltzmann constant, $T$ the temperature, $M$ the equivalent mass of the resonator, $\mathrm{f}_{\mathrm{r}}$ its eigen frequency, and $\mathrm{Q}$ its quality factor.

\section{2) Closed loop detection noise}

The main noise source for the closed loop detection originates in the oscillator phase noise. Its power spectral density can be derived from the Leeson equation [11]:

$$
S_{\phi}(\delta f)=\frac{F k T}{P_{0}}\left(1+\frac{f_{r}^{2}}{4 Q^{2} \delta f^{2}}\right)\left(1+\frac{f_{c}}{\delta f}\right) \quad\left(\operatorname{rad}^{2} / \mathrm{Hz}\right),
$$

where $\delta f=f-f_{r}$ is the carrier offset frequency, $F$ is the noise factor of the oscillator feedback circuit, $f_{c}$ is the flicker phase noise corner of the oscillator feedback circuit, $P_{0}$ the power through the resonator.

As detailed in [12], the corresponding frequency noise power spectral density can be expressed as:

$$
S_{F}(\delta f)=\delta f . S_{\phi}(\delta f) \quad(15) .
$$

As explained in section II.B, the photoacoustic force is calculated from the eigen frequency variation of the oscillator. In the same way, the force noise power spectral density of the closed loop detection oscillator can be calculated from frequency noise, taking into account (8):

$$
F_{\text {closed_loop }}=\left(S_{F} \times \frac{F_{P A}}{\Delta f_{r}}\right)^{2}(16)
$$

Finally with equation (16), (14) and (15) one can obtain:

$$
\begin{gathered}
F_{\text {closed loop }}(\delta f)= \\
\left(\frac{2 Q F_{\mathrm{pz}}}{f_{r}}\right)^{2} \delta f^{2}\left(1+\frac{f_{r}^{2}}{4 Q^{2} \delta f^{2}}\right)\left(1+\frac{f_{c}}{\delta f}\right) \frac{F k T}{P_{0}}\left(\mathrm{~N}^{2} / \mathrm{Hz}\right)(1
\end{gathered}
$$

\section{3) Noise comparison}

In order to compare noise levels of the two detection schemes, the noise power spectral density is computed from (13) and (16) and shown in Fig. 4. The two configurations are compared: the open loop detection scheme (red line) and the closed loop detection scheme (blue line) working with the same resonator used for experimental results. The parameters of the resonator and the oscillator circuit taken into account are listed in Table 1.

Considering this resonator, the noise of this new technique is worse than the noise of the open loop scheme. This drawback can be overcome by decreasing the piezoelectric actuation force as can be deduced from (16). This can be done by adjusting electrodes configuration on the resonator to decrease actuation force and increase piezoelectric detection efficiency.

Table 1: Parameters of the resonator and oscillator circuit used for calculations and experimental results.

\begin{tabular}{|c|c|c|}
\hline Parameter & Unit & Value \\
\hline Q factor & & 8000 \\
\hline Motional resistance & $\mathrm{M} \Omega$ & 3 \\
\hline Resonator eigen frequency $\left(\mathrm{f}_{\mathrm{r}}\right)$ & $\mathrm{Hz}$ & 21000 \\
\hline $\begin{array}{c}\text { Resonator equivalent mass } \\
(\mathrm{M})\end{array}$ & $\mathrm{kg}$ & $1.410^{-4}$ \\
\hline $\begin{array}{c}\text { Oscillator Noise Factor }(\mathrm{F}) \\
\text { Oscillator flicker phase noise } \\
\text { corner frequency }(\mathrm{fc})\end{array}$ & $\mathrm{Hz}$ & 1 \\
\hline Oscillator power $\left(\mathrm{P}_{0}\right)$ & $\mathrm{W}$ & $8.310^{-10}$ \\
\hline Piezoelectric force $\left(\mathrm{F}_{\mathrm{pz}}\right)$ & $\mathrm{N}$ & $10^{-6}$ \\
\hline Photoacoustic force $\left(\mathrm{F}_{\mathrm{pa}}\right)$ & $\mathrm{N}$ & $10^{-13}$ \\
\hline
\end{tabular}




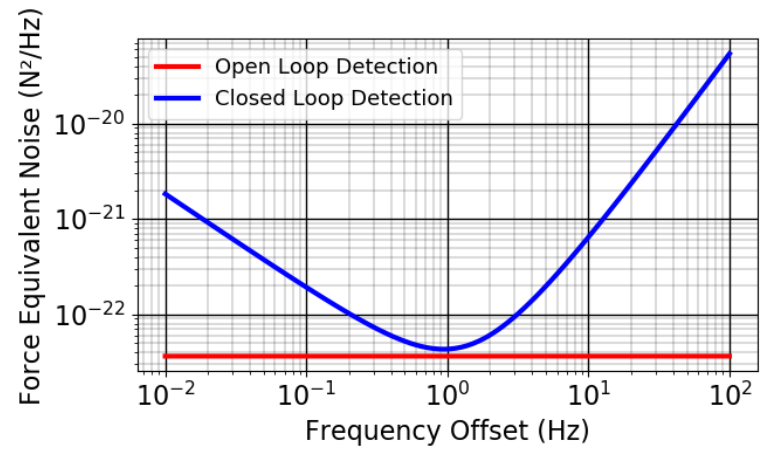

Figure 4: Force equivalent noise power spectral density for open loop detection and closed loop detection, calculated with the parameters of Table 1.

\section{EXPERIMENTAL RESULTS}

\section{A. Experimental set-up}

The experimental scheme is shown in Fig. 5. The resonator is placed in a hermetic chamber filled with $\mathrm{C}_{2} \mathrm{H}_{2}$ diluted at $1 \%$ in air at atmospheric pressure and room temperature. The laser source is a telecom laser diode, its wavenumber is set at 6,490.05 $\mathrm{cm}^{-1}$ corresponding to a rovibrational transition of $\mathrm{C}_{2} \mathrm{H}_{2}$ as shown in Fig. 6. There is no interference from residual water vapor at this wavelength.

The oscillator circuit is an analog self-sustained oscillator, its output signal controls a waveform generator that enables to control the phase shift of the photoacoustic branch, as well as the waveform and the amplitude of the Mach-Zehnder input for amplitude modulation of the laser radiation. The oscillator frequency shift, proportional to the $\mathrm{C}_{2} \mathrm{H}_{2}$ concentration, is measured with a frequency counter (Agilent 53132A).

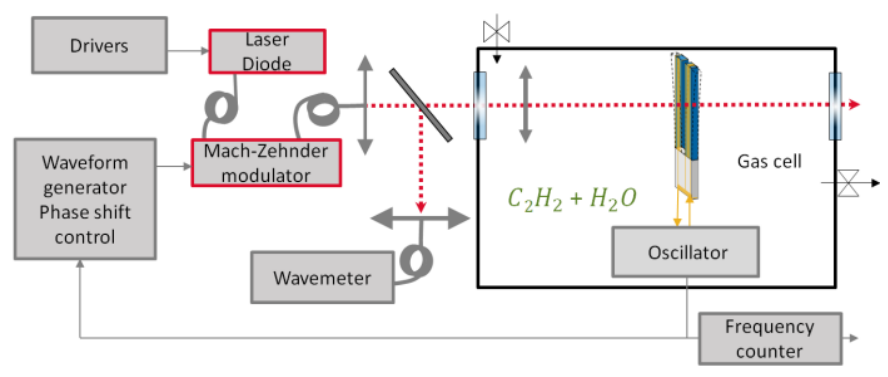

Figure 5: Experimental set-up to test the closed loop detection scheme.

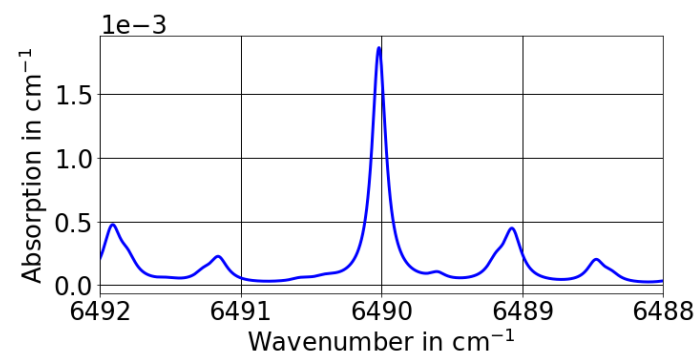

Fig. 6: Calculated absorption of $\mathrm{C}_{2} \mathrm{H}_{2}$ at 1 percent concentration at standard atmospheric conditions, using a Voigt broadening model and the HITRAN database
The tuning fork resonator used for the experiment is shown in Fig. 7. It has been designed and realized at ONERA for QEPAS operation [13]. In order to increase the eigen frequency variations over photoacoustic actuation force, the piezoelectric force must be kept as small as possible, as shown in (8). For this purpose only one third of the actuation electrodes have been used for piezoelectric actuation. The parameters of the resonator and the oscillator circuit used for the experiment are shown in Table 1.

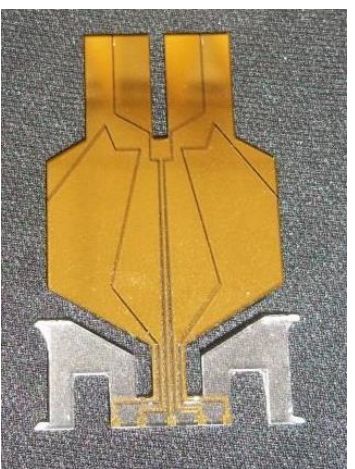

Figure 7: Tuning fork resonator used for the experiment.

\section{B. Experimental results}

\section{1) Setting the phase shift}

In order to maximize the eigen frequency variation over the photoacoustic actuation force, the phase shift $\Phi_{\text {elec }}$ must be adjusted with the phase shifter circuit to set a phase quadrature between the photoacoustic force and the piezoelectric force, as shown in (2). For that purpose the oscillator frequency variation $\Delta f_{r}$ is measured over the phase variation of the phase shifter circuit $\Phi_{\text {elec }}$ as shown in Fig. 8. The phase values corresponding to the minimum and maximum frequency variations are measured:

$$
\begin{gathered}
\left.\Phi_{\text {elec }}\right|_{\text {min }}=60^{\circ} \\
\left.\Phi_{\text {elec }}\right|_{\text {max }}=\left.\Phi_{\text {elec }}\right|_{\text {min }}+180^{\circ}=240^{\circ}
\end{gathered}
$$

These values are measured at the beginning of the experiment, and do not need to be measured again, provided that the sound velocity in the gas does not change.

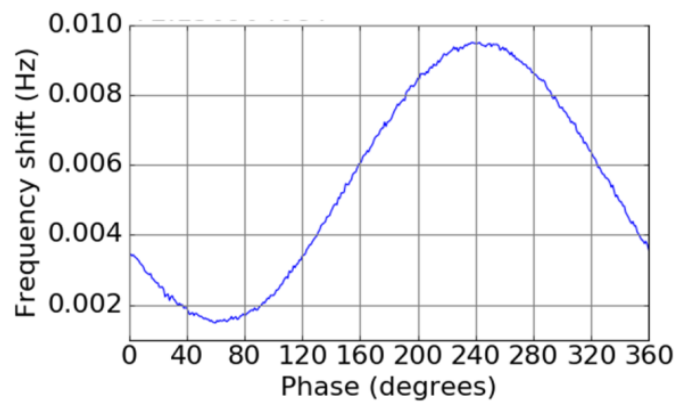

Figure 8: Resonator eigen frequency shift over phase of the phase shifter circuit.

\section{2) Frequency shift measurement}


Fig. 9 shows the oscillator frequency shift over laser operation. When the laser is on, the frequency increases when the phase shift is set to $\left.\Phi_{\text {elec }}\right|_{\text {min }}$ (red line), and decreases when the phase shift is set to $\Phi_{\text {elec }} I_{\max }$ (blue line), as described in Eqs. 9 and 10. An additional frequency decrease over time is common to the two oscillator frequencies: it corresponds to the frequency drift with temperature. This temperature sensitivity can be cancelled by computing the difference of the two frequencies, as shown in Fig. 9 and described in (11).

The measurement time used in Fig. 9 and 10 is $1 \mathrm{~s}$, the measurements being taken alternatively at $0^{\circ}$ and $180^{\circ}$ phase shifts. 15 measurements are taken alternatively while the laser is off and on. Measurements have also been performed down to $50 \mathrm{~ms}$ in order to validate the fast measurements ability of the closed loop detection.

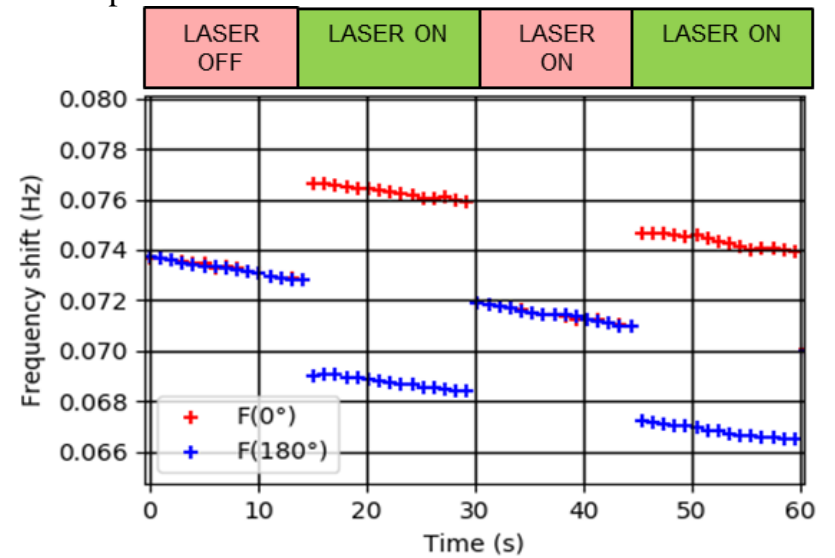

Figure 9: Resonator eigen frequency shift vs. laser on/off. The frequency shift is positive when the additional phase shift is null (red line), whereas it is negative when the additional phase shift is $180^{\circ}$ (blue line). In addition to the photoacoustic signal frequency sensitivity, the frequency also shifts over time due to temperature variations.

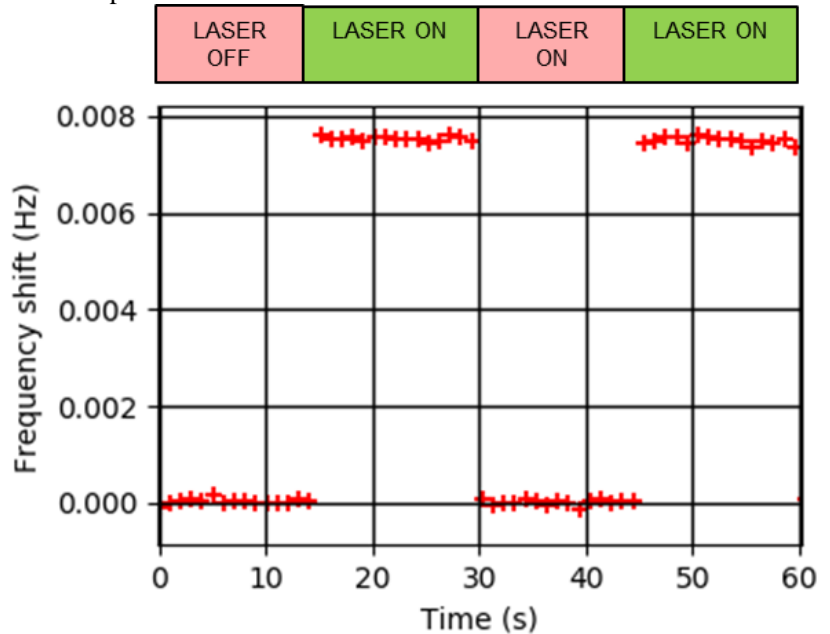

Figure 10: Differential eigen frequency shift vs. laser on/off. The common mode frequency variation over temperature has been cancelled by the differential measurement.

\section{3) Noise equivalent relative absorption measurement}

The relative absorption noise can be related to the Allan deviation of the oscillator frequency over measurement time:

$$
\sigma_{r a}(\tau)=\frac{K}{\alpha} \sigma_{f}(\tau)
$$

where $\sigma_{f}(\tau)$ is the oscillator frequency Allan deviation measured with different gate times $\tau, \alpha$ is the absorption coefficient at the line center calculated from HITRAN database and shown in Fig. $5\left(\alpha=1.910^{-3} \mathrm{~cm}^{-1}\right)$, and $K$ is the ratio between the gas absorption and the resulting differential oscillator frequency variation. $K$ has been measured experimentally in the case of the experiment described in section $\mathrm{A} /$ for $1 \% \mathrm{C}_{2} \mathrm{H}_{2}$ and the oscillator parameters of Table $1, K=0.24 \mathrm{~cm}^{-1} / \mathrm{Hz}$.

Relative absorption noise is plotted in Fig. 11, and measurements with integration time of $100 \mathrm{~ms}$ is shown in Fig. 12. The sensitivity of a photoacoustic detector can be qualified by a figure of merit, called normalized noise equivalent absorption (NNEA). It represents the minimum detectable absorption, independently from the laser power, the electrical bandwidth and the absorption coefficient of the gas. It is defined as follows:

$$
N N E A=\frac{P_{L} \alpha}{S N R \sqrt{B W}}
$$

where $P_{L}$ is the laser power $(17 \mathrm{~mW})$, SNR is the signal to noise ratio calculated from relative absorption Allan deviation, and BW is the measurement bandwidth, ie the inverse of the Allan deviation integration time. The calculated NNEA is:

$$
\mathrm{NNEA}=310^{-7} \mathrm{~W} \cdot \mathrm{cm}^{-1} / \sqrt{\mathrm{Hz}} \text {. }
$$

This value, obtained by this first experiment of QEPAS closed loop detection, is worse than the NNEA obtained with open loop lock-in detection. This is in agreement with theoretical model of noise shown in Fig. 4. The SNR can be improved by improving the electrodes design. Indeed, from (8) it is clear that the piezoelectric actuation force must be as low as possible to increase the measured frequency shift. One way to do this is to make the actuation electrodes very small, enabling to use the resonator surface available to design longer detection electrodes, and then to obtain an improved signal to noise ratio.

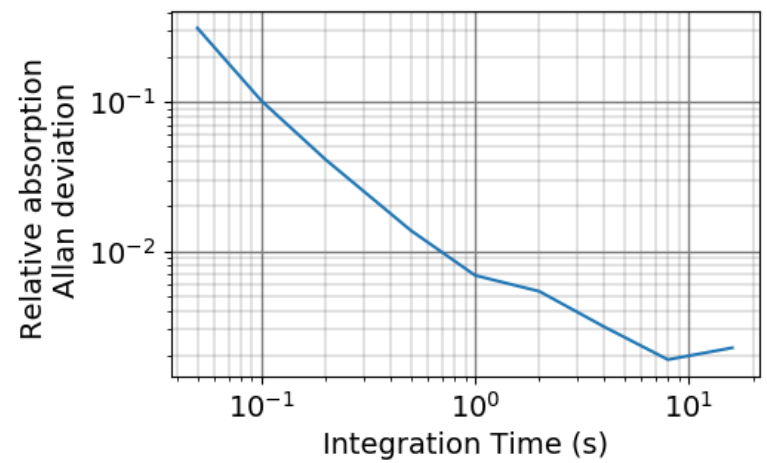

Figure 11: Measured relative absorption Allan deviation over integration time. 


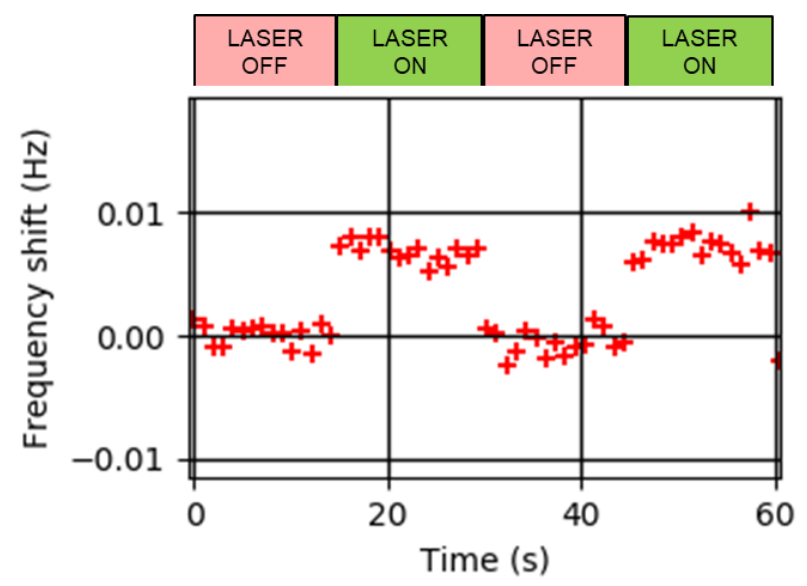

Figure 12: Differential frequency shift vs. laser on/off for integration time of $1 \mathrm{~s}$ (top) and $100 \mathrm{~ms}$ (bottom) for $1 \% \mathrm{C}_{2} \mathrm{H}_{2}$ and the oscillator parameters of Table 1.

\section{CONCLUSION}

A new active detection scheme for QEPAS has been described; a theoretical model has been shown and validated by experimental measurements. This new detection scheme enables fast and precise measurements. Fast measurements down to $50 \mathrm{~ms}$ integration time have been performed and confirm the precise and thermal drift free measurements. Future work will focus on the signal to noise ratio improvement by optimizing the resonator and its electrodes scheme, and by implementing the detection scheme in synchronous digital electronics on FPGA.

\section{ACKNOWLEDGMENT}

The project was partially funded by the FCS Campus ParisSaclay under grant 2014-0455I (MICSI).

[1] BELL, Alexander Graham. Upon the production of sound by radiant energy. Gibson Brothers, printers, 1881.

[2] Spagnolo, V., Patimisco, P., Pennetta, R., Sampaolo, A., Scamarcio, G., Vitiello, M. S., \& Tittel, F. K. (2015). THz Quartz-enhanced photoacoustic sensor for $\mathrm{H}_{2} \mathrm{~S}$ trace gas detection. Optics Express, 23(6), 7574-7582.

[3] Kosterev, Anatoliy A., et al. "Quartz-enhanced photoacoustic spectroscopy." Optics letters 27.21 (2002): 1902-1904.

[4] Petra, N., Zweck, J., Kosterev, A. A., Minkoff, S. E., \& Thomazy, D. (2009). Theoretical analysis of a quartz-enhanced photoacoustic spectroscopy sensor. Applied Physics B, 94(4), 673-680.

[5] Aoust, G., Levy, R., Raybaut, M., Godard, A., Melkonian, J. M., \& Lefebvre, M. (2017). Theoretical analysis of a resonant quartz-enhanced photoacoustic spectroscopy sensor. Applied Physics B, 123(2), 63.

[6] Patimisco, P., Scamarcio, G., Tittel, F. K., \& Spagnolo, V. (2014). Quartzenhanced photoacoustic spectroscopy: a review. Sensors, 14(4), 61656206.

[7] Aoust, G., Levy, R., Raybaut, M., Melkonian, J. M., Bourgeteau, B., Godard, A., \& Lefebvre, M. (2016, June). Optimal tuning fork for quartz enhanced photoacoustic spectroscopy. In 2016 Conference on Lasers and Electro-Optics (CLEO) (pp. 1-2). IEEE.

[8] Zheng, H., Dong, L., Yin, X., Liu, X., Wu, H., Zhang, L., Ma, W., Yin, W., \& Jia, S. (2015). Ppb-level QEPAS NO2 sensor by use of electrical modulation cancellation method with a high power blue LED. Sensors and Actuators B: Chemical, 208, 173-179.

[9] Mordmüller, M., Köhring, M., Schade, W., \& Willer, U. (2015). An electrically and optically cooperated QEPAS device for highly integrated gas sensors. Applied Physics B, 119(1), 111-118.
[10] Wu, H., Dong, L., Zheng, H., Yu, Y., Ma, W., Zhang, L., Yin, W., Xiao L., Jia, S., \& Tittel, F. K. (2017). Beat frequency quartz-enhanced photoacoustic spectroscopy for fast and calibration-free continuous tracegas monitoring. Nature communications, 8, 15331.

[11] Rubiola, E. (2009). Phase noise and frequency stability in oscillators. Cambridge University Press.

[12] Levy, R., \& Gaudineau, V. (2010, June). Phase noise analysis and performance of the vibrating beam accelerometer. In Frequency Control Symposium (FCS), 2010 IEEE International (pp. 511-514). IEEE.

[13] Aoust, G., Levy, R., Verlhac, B., \& Le Traon, O. (2018). Ultra high quality factor resonators operated in fluids. Sensors and Actuators A: Physical, 269, 569-573.8, 15331.

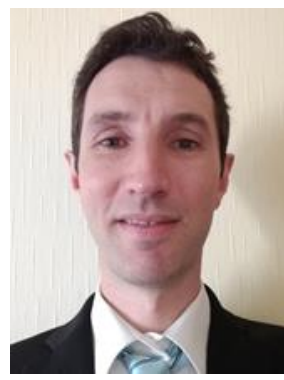

Raphaël Levy was born in Paris in 1979. $\mathrm{He}$ got his Ph.D. in Microtechnology engineering from University of ParisSaclay in 2005. Since 2007, he is a research engineer at the French Aerospace Lab (ONERA). His current research interests include High performance MEMS sensors design and associated microtechnologies. He is now Head of Sensors and Micro/Nano

Technology Unit.

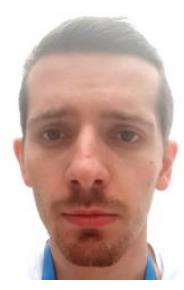

Maxime Duquesnoy received an Engineering degree from ENSICAEN in 2017 in the field of electronics and sensors. He is currently Ph.D. candidate at Mirsense (Palaiseau, France) and at ONERA (Palaiseau, France) on photoacoustic spectroscopy based on quartz tuning forks.

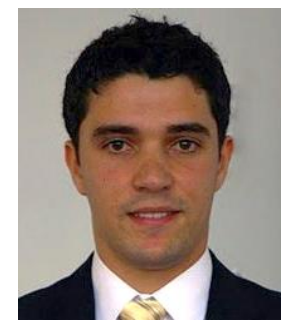

Guillaume Aoust received the M.Sc. degree from Ecole Polytechnique (Palaiseau, France) in 2011 in the field of lasers and optics, as well as the M.Sc. degree from the Ecole Nationale des Ponts et Chaussées (Champ-sur-Marne) in 2013 in the field of acoustics and mechanics. He obtained his Ph.D. from the Paris Saclay University in 2016, for his work entitled "Development of infrared sources and quartz resonators for photoacoustic spectroscopy" and performed at The French Aerospace Lab (ONERA, France) and in Capasso Group (Harvard University). He is currently a R\&D scientist at MirSense company in charge of the design and manufacture of photoacoustic based trace gas detectors. 


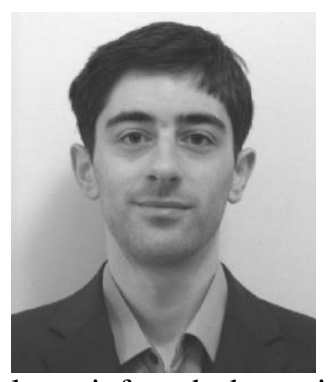

Jean-Michel Melkonian has a degree in engineering from Institut d'Optique and a Ph.D. in physics from Ecole Polytechnique. He is working at Onera - The French Aerospace Lab, on optical sensing of hazardous and greenhouses gases, for space, defense, and civilian applications. His current research interests include photoacoustic and laser infrared absorption spectroscopy, remote sensing using lidars, and the development of mid infrared laser and optical parametric sources.

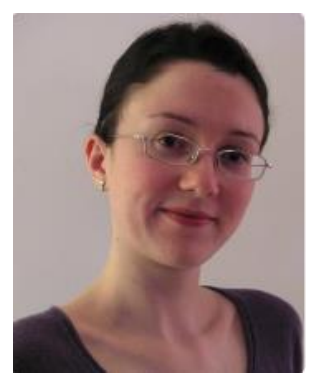

Myriam Raybaut graduated from Institut d'Optique Graduate School in 2003, and got her PhD from Université Paris XI in 2006, working on quasiphase matching techniques in semiconductor materials. Since 2007, she has been working as a research scientist at Onera. So far, her research interests have included specific laser and optical parametric sources development, as well as local and remote gas sensing instrumentation development. 